\title{
Evaluation of irritable bowel syndrome prevalence in medical students
}

\author{
Samin Maghsoudi ${ }^{\circledR}$, Azam Teimouri ${ }^{2,3^{*}}$ (๑) \\ ${ }^{1}$ Faculty of Medicine, Isfahan University of Medical Sciences, Isfahan, Iran \\ ${ }^{2}$ Department of Gastroenterology, Isfahan University of Medical Sciences, Isfahan, Iran \\ ${ }^{3}$ Isfahan Gastroenterology and Hepatology Research Center, Isfahan University of Medical Sciences, Isfahan, Iran
}

\section{Correspondence to:}

Azam Teimouri, E-mail: a

teimouri@med.mui.ac.ir

Received: 30 Apr. 2021 Accepted: 25 June 2021 ePublished: 20 July 2021

Keywords: Irritable bowel syndrome, Medical student Prevalence

\begin{abstract}
Introduction: Irritable bowel syndrome (IBS) is a functional gastrointestinal disorder and requires early diagnosis due to its negative impacts on the quality of life. According to the lifestyle pattern of medical students, IBS has a significant prevalence in these individuals.

Objectives: The present study aims to investigate the prevalence and factors associated with IBS in medical students.

Patients and Methods: This cross-sectional study has been conducted on 100 medical students studying at Isfahan University of Medical Sciences in 2019-2020. The Rome IV diagnostic criteria were administered to diagnose IBS and individuals were divided into four groups of constipation-dominant (IBS-C), diarrheadominant (IBS-D), mixed type (IBS-M) and unspecified (IBS-U). The association of studying grade, gender and residence with IBS was evaluated.

Results: The mean age of the students was $23.2 \pm 4.8$ years, including $68(68 \%)$ women. According to Rome -IV criteria, 24 (24\%) ones were diagnosed with IBS. The IBS cases were dominantly in IBS-C group $(54.16 \%)$. IBS was not associated with gender $(P=0.498)$, educational grade $(P=0.661)$ and type of residence $(P=0.562)$ in the studied medical students.

Conclusion: According to the present study, the prevalence of IBS was $24 \%$ among medical students, which was higher among women. Most patients had IBS-C, but IBS did not show a statistically significant relationship with gender, educational grade and type of residence.
\end{abstract}

Citation: Maghsoudi S, Teimouri A. Evaluation of irritable bowel syndrome prevalence in medical students. J Prev Epidemiol. 2021;6(2):e21. doi: $10.34172 /$ jpe.2021.21

\section{Introduction}

Irritable bowel syndrome (IBS) is among the functional gastrointestinal disorder and one of the most common outpatient clinical diagnoses (1). Epigastric discomfort or abdominal pain with alteration in bowel habits with no anatomical or histopathological change in gastrointestinal system are the hallmarks of IBS (2).

The prevalence of IBS considerably varies around the world, which is relatively related to the diagnostic criteria. According to epidemiological studies, $5.7 \%-34 \%$ of different populations are struggling with IBS (3). The studies in the western countries represent $17 \%-22 \%$ of IBS prevalence, while a wide range of $2.3 \&-34 \%$ in Asian countries has been noted $(4,5)$.

The pathogenesis of IBS remained unknown and various biological, psychological and social factors such as gender, dietary regimen, lifestyle, stress, posttraumatic stress disorder and psychological disturbances have been stated $(2,6,7)$.

\begin{abstract}
Key point
Irritable bowel syndrome (IBS) is a functional gastrointestinal disorder that affects daily performance and quality of life negatively. Medical students are at higher risks for IBS development due to the stressful nature of their life and occupation. Providing preventive strategies to minimize the risk of IBS in this critical population can help better performance of medical students that are future physicians.
\end{abstract}

Medical students experience numerous factors that make them prone to IBS. This critical population faces distressful conditions, including high levels of emotions, heavy examinations, occupational competition, improper dietary regimen and lifestyle, and significant responsibilities to the patients (8). All these factors clarify that the prevalence of IBS is high among medical students $(3,9,10)$.

IBS complications are considerably noteworthy as it impairs quality of life; reduce educational success, increases

Copyright ( 2021 The Author(s); Published by Society of Diabetic Nephropathy Prevention. This is an open-access article distributed under the terms of the Creative Commons Attribution License (http://creativecommons.org/licenses/by/4.0), which permits unrestricted use, distribution, and reproduction in any medium, provided the original work is properly cited. 
absences at university and work. Nevertheless, the present medications are limited, and paucity of knowledge is available for therapeutic approaches for IBS (11).

\section{Objectives}

The current study is among the restricted ones in Iran assessing the prevalence and symptoms of IBS in medical students of Isfahan University of Medical Sciences.

\section{Patients and Methods}

\section{Study design}

The current cross-sectional study has been conducted on 100 medical students of Isfahan university of medical sciences from January to July 2020.

Studying at any stage of medicine at Isfahan university of medical sciences was the inclusion criterion. Reluctance to participate in the study and the presence of any gastrointestinal alarm signs (dysphagia, odynophagia, anorexia, excessive weight loss, and nocturnal abdominal pain), education drop out, inflammatory bowel disease, positive family history of gastric or large intestine cancers were considered as the unmet criteria. Over 20\% defect in filled questionnaires was the exclusion criterion.

The studied population entered into the study through convenience sampling.

The demographic information, including age, gender and residence (personal house or dormitory) was entered into the study checklist.

\section{Irritable bowel syndrome}

Rome IV criteria were administered to diagnose IBS. The patients with recurrent abdominal pain for at least once a week since the previous three months with the least two of the symptoms below were determined as IBS:

- Be related to defecation (probably improved by defecation, deteriorated or with no change),

- Be related to daily defecation frequency,

- Be associated with fecal appearance (12).

Besides, based on the dominant manifestation, IBS was divided into 4 groups of constipation dominant (IBS-C), diarrhea dominant (IBS-D), mixed (IBS-M) and unclassified (IBS-U). Therefore, the IBS subtype was determined based on the dominant presentations of bowel habits in days with abnormal bowel movements. Accordingly, IBS-C and IBS-D are defined if the predominant complaint of the patient in the days with bowel irritability is constipation or diarrhea, respectively. Mixed type is indicated by concurrent presentation of diarrhea and constipation, while at least one-fourth of the defecations are diarrhea. Eventually, if none of the above criteria is met, the patient is classified as IBS-U (13). The sensitivity and specificity of Rome IV for IBS diagnosis are $62.7 \%$ and $97.1 \%$, respectively (14).

\section{Data analysis}

The obtained data were imported to the Statistical Package for the Social Sciences (SPSS) (IBM Crop. 2019. IBM SPSS Statistics for Windows, version 26.0. NY, EUA). The descriptive data were presented as frequencies, percentages, mean and standard deviation. Chi-square or Fishers' exact tests were administered to compare the categorical parameters. Independent student $t$ test was applied to compare quantitative data. $P$ value of less than 0.05 was considered as the significant level.

\section{Results}

One hundred and six medical students were included in the study among which 100 ones completed the questionnaires. Mean age of the studied population was $23.2 \pm 4.8$ years old (range: 18-28 years) with predominance of females gender (68\%). Most of the participants were residents of dormitory (78\%) and studied in physiopathology 64\%), basic sciences (21\%) and internship (15\%) stages, respectively.

Based on Rome-IV, 24 students, including 18 (75\%) females had IBS. The most common complaint of the patients was abdominal pain improvement by defecation (91\%). The detailed information is presented in Table 1.

Besides, most of the studied patients were categorized in constipation dominant (IBS-C) group with 13 ones (54.16\%). The other categories of IBS, included IBS-D, IBS-M and IBS-U with five (20.83\%), four (16.67\%) and three $(12.5 \%)$ cases, respectively. Based on Table 2, IBS was not associated with gender $(P=0.498)$, studying stage $(P=0.661)$ and residence $(P=0.562)$ in the medical students.

\section{Discussion}

Functional gastrointestinal disorders are among the most common etiologies of outpatient clinical referrals and the patient refer to the physicians because of impaired quality of life. IBS is one of the prevalent gastrointestinal disorders that despite inadequate knowledge about its etiology is remarkably associated with lifestyle and psychological disturbances (1). The lifestyle, irregular sleep time, permanent stress and dietary regimen make medical students at increased risk for functional gastrointestinal disorders, IBS in particular (15).

In this study, 100 medical students were assessed according to Rome IV criteria among which 24 ones (24\%) were diagnosed with IBS. Population-based studies revealed diverse prevalence of IBS in different communities $(16,17)$. Nevertheless, the studies are aligned with each other that IBS is more prevalent among medical students than the general population (15). Naeem and colleagues applied Rome III criteria and presented 28.3\% of medical students were involved with IBS (2). This rate accounted for $33.3 \%$ of the medical students who participated in Liu et al study conducted in the East of Asia (1). In line with these studies, the other ones presented the frequencies of $20.6 \%$ in Lebanon (18), 20.6\% in Canada (19), $22.2 \%$ (20) and 35\% (10) in Japan. On the other hand, the studies in Nigeria (21) and Malaysia (22) presented less 
Table 1. The symptoms of irritable bowel syndrome in the medical students

\begin{tabular}{|c|c|c|c|}
\hline Irritable bowel syndrome symptoms & $\begin{array}{l}\text { Female } \\
\text { No. }(\%)\end{array}$ & $\begin{array}{l}\text { Male } \\
\text { No. (\%) }\end{array}$ & $\begin{array}{l}\text { Total } \\
\text { No. }(\%)\end{array}$ \\
\hline Change in the frequency of defecation & $14(73.6)$ & $5(83.3)$ & $19(76)$ \\
\hline Change in fecal appearance & $16(88.8)$ & $5(83.3)$ & $21(84)$ \\
\hline Defecation urgency & $13(72.2)$ & $4(66.6)$ & $17(68)$ \\
\hline Change in abdominal pain by defecation & $17(94.4)$ & $5(83.3)$ & $22(91)$ \\
\hline
\end{tabular}

Table 2. The association of irritable bowel syndrome and demographic characteristics of the medical students

\begin{tabular}{|c|c|c|c|c|}
\hline \multirow{2}{*}{ Variables } & \multirow{2}{*}{ Total $(n=100)$} & \multicolumn{2}{|c|}{ Irritable bowel syndrome } & \multirow{2}{*}{$P$ value } \\
\hline & & Healthy $(n=76)$ & Patients $(n=24)$ & \\
\hline \multicolumn{5}{|l|}{ Gender } \\
\hline Female & $69(69 \%)$ & $51(67.4 \%)$ & $18(75 \%)$ & \multirow{2}{*}{0.498} \\
\hline Male & $31(31 \%)$ & $25(32.6 \%)$ & $6(25 \%)$ & \\
\hline \multicolumn{5}{|l|}{ Educational level } \\
\hline Basic sciences & $21(21 \%)$ & $18(23.9 \%)$ & $3(12.5 \%)$ & \multirow{3}{*}{0.661} \\
\hline Physiopathology & $64(64 \%)$ & $46(59.8 \%)$ & $18(75 \%)$ & \\
\hline Internship & $15(15 \%)$ & $12(16.3 \%)$ & $3(12.5 \%)$ & \\
\hline \multicolumn{5}{|l|}{ Residence } \\
\hline Native & $22(22 \%)$ & $16(16.7 \%)$ & $6(25 \%)$ & \multirow{2}{*}{0.562} \\
\hline Dormitory & $78(78 \%)$ & $60(78.3 \%)$ & $18(75 \%)$ & \\
\hline
\end{tabular}

than $15 \%$ in their medical students. However, the current study is similar to most of the other studies in this area; it seems that the educational protocols, daily working time, psychological burden of working and lifestyle are the factors associated with the incidence of IBS in medical students over the world. The administered instrument to diagnose IBS or include the participant is the other probable factors affecting the measured prevalence.

The most common diagnostic symptom for IBS in our study was the change in pain severity after defecation. This finding is consistent with the other studies that presented change in fecal appearance, frequency of defecation and urgency in defecation following the above one $(1,15,23)$.

Most of the patients were categorized in the IBS-C group which is in agreement with the other studies conducted in Iran; a fact that probably occurs due to the type of dietary regimen and culture of eating in $\operatorname{Iran}(9,24,25)$. All the studies have unanimously reported the predominance of IBS incidence in females and IBS-C. In addition, constipation is more common in females. This association is to the extent that female gender is defined as a prognostic factor for IBS-C $(24,26)$.

The other studies declared diverse frequencies of IBS categories. Most of the medical students in China had IBS-M (1). Jemilohun et al represented IBS-M in $57.8 \%$ of their study on general population (21).

In our study, females were more affected by IBS; however, this difference was not statistically significant. The higher rate of IBS in female gender is consistent with most of the other population-based studies that presented approximately female to male proportion of 2:1 $(1,27)$.

Besides, in the current report, IBS incidence increased by upgrading of educational level, but not significantly.
This increase in the incidence seems to occur because of impaired sleep times, irregularity and high speed of eating meals, turning to ready meals and increased levels of stress due to responsibilities of visiting the patients. This association has been presented by the other studies on medical students that represented a direct relation between level of stress and IBS $(1,28)$. Ibrahim et al reported the level of stress as the factor associated with IBS and deterioration of the condition by educational upgrading (29).

We found no association between IBS and residence of medical students. Most of the previous studies presented increased risk of IBS development in students living in a dormitory. They found that inappropriate dietary habits, lifestyle and sleep pattern, and the stress due to struggling with a new community and sense of being support-less are the factors associated with IBS in the students living in dormitories $(1,19,28)$.

\section{Conclusion}

According to the current study, IBS had the prevalence of $24 \%$ in medical students. Most of the patients had IBS-C. This syndrome was not associated with gender, stage of studying, and residence of the medical students.

\section{Limitations of study}

The most significant limitation of this study is not to include the patients using block sampling which occurred due to inappropriate cooperation of the students studying at higher stages. Although there was no difference in the distribution of IBS in different stages of medical studying, this irregular distribution may have led to a potential bias and should be considered in further studies. The small sample population is another limitation of this 
study. Further studies by consideration of more detailed confounders affecting sleep quality are recommended. In addition, we have not evaluated the course of study as a potential risk factor for sleep dysfunction among the studied population.

\section{Acknowledgments}

The authors want to present their most appreciation to Dr. Ali Safaei for his efforts in the conduction of this study. The current investigation was performed according to proposal encoded as IR.MUI.MED.REC.1399.1091 by the Ethics Committee of Isfahan University of Medical Sciences.

\section{Authors' contribution}

SM and AT were the principal investigators of the study. SM and AT were included in preparing the concept and design. SM and AT revisited the manuscript and critically evaluated the intellectual contents. All authors participated in preparing the final draft of the manuscript, revised the manuscript and critically evaluated the intellectual contents. All authors have read and approved the content of the manuscript and confirmed the accuracy or integrity of any part of the work.

Conflicts of interest

The authors declare that they have no competing interests.

\section{Ethical issues}

The research followed the tenets of the Declaration of Helsinki. The Ethics Committee of Isfahan University of Medical Sciences approved this study. The institutional ethical committee at Isfahan University of Medical Sciences approved all study protocols (IR.MUI.MED.REC.1399.1091). Accordingly, written informed consent was taken from all participants before any intervention. This study was extracted from M.D thesis of Samin Maghsoudi at this university. Moreover, ethical issues (including plagiarism, data fabrication, double publication) have been completely observed by the authors.

\section{Funding/Support}

The current paper has been sponsored by Isfahan University of Medical Sciences. No further sponsorship has been administered.

\section{References}

1. Liu $Y$, Liu L, Yang $Y$, He $Y$, Zhang $Y$, Wang $M$, Chen $S$, Yao $S$. A school-based study of irritable bowel syndrome in medical students in beijing, china: prevalence and some related factors. Gastroenterol Res Pract. 2014;2014:124261. doi: 10.1155/2014/124261.

2. Naeem SS, Siddiqui EU, Kazi AN, Memon AA, Khan ST, Ahmed B. Prevalence and factors associated with irritable bowel syndrome among medical students of Karachi, Pakistan: a cross-sectional study. BMC Res Notes. 2012;5:255. doi: 10.1186/1756-0500-5-255.

3. Abdulmajeed A, Rabab MA, Sliem HA, Hebatallah NE. Pattern of irritable bowel syndrome and its impact on quality of life in primary health care center attendees, Suez governorate, Egypt. Pan Afr Med J. 2011;9:5. doi: 10.4314/pamj.v9i1.71177.

4. Shen L, Kong H, Hou X. Prevalence of irritable bowel syndrome and its relationship with psychological stress status in Chinese university students. J Gastroenterol Hepatol. 2009;24:188590. doi: 10.1111/j.1440-1746.2009.05943.x.

5. Talley NJ, Zinsmeister AR, Van Dyke C, Melton LJ 3rd. Epidemiology of colonic symptoms and the irritable bowel syndrome. Gastroenterology. 1991;101:927-34. doi:

\subsection{6/0016-5085(91)90717-y.}

6. Anbardan SJ, Daryani NE, Fereshtehnejad SM, Taba Taba Vakili S, Keramati MR, Ajdarkosh H. Gender Role in Irritable Bowel Syndrome: A Comparison of Irritable Bowel Syndrome Module (ROME III) Between Male and Female Patients. J Neurogastroenterol Motil. 2012;18:70-7. doi: 10.5056/ jnm.2012.18.1.70.

7. Basandra S, Bajaj D. Epidemiology of Dyspepsia and Irritable Bowel Syndrome (IBS) in Medical Students of Northern India. J Clin Diagn Res. 2014;8:JC13-6. doi: 10.7860/ JCDR/2014/10710.5318.

8. Jung HJ, Park MI, Moon W, Park SJ, Kim HH, Noh EJ, Lee G], Kim JH, Kim DG. Are Food Constituents Relevant to the Irritable Bowel Syndrome in Young Adults? - A Rome III Based Prevalence Study of the Korean Medical Students. J Neurogastroenterol Motil. 2011;17:294-9. doi: 10.5056/ jnm.2011.17.3.294.

9. Mansour-Ghanaei F, Fallah M, Heidarzadeh A, Jafarshad R, Joukar F, Ghasemipour R, et al. Prevalence and characteristics of irritable bowel syndrome (IBS) amongst medical students of Gilan Northern Province of Iran. MEJDD. 2009;1:100-5.

10. Okami Y, Kato T, Nin G, Harada K, Aoi W, Wada S, et al. Lifestyle and psychological factors related to irritable bowel syndrome in nursing and medical school students. J Gastroenterol. 2011;46:1403-10. doi: 10.1007/s00535-011-0454-2.

11. Nellesen D, Yee K, Chawla A, Lewis BE, Carson RT. A systematic review of the economic and humanistic burden of illness in irritable bowel syndrome and chronic constipation. J Manag Care Pharm. 2013;19:755-64. doi: 10.18553/ jmcp.2013.19.9.755.

12. Schmulson MJ, Drossman DA. What Is New in Rome IV. J Neurogastroenterol Motil. 2017;23:151-163. doi: 10.5056/ jnm16214.

13. Drossman DA, Hasler WL. Rome IV-Functional GI Disorders: Disorders of Gut-Brain Interaction. Gastroenterology. 2016;150:1257-61. doi: 10.1053/j.gastro.2016.03.035

14. Palsson OS, Whitehead WE, van Tilburg MA, Chang L, Chey W, Crowell MD, et al. Rome IV Diagnostic Questionnaires and Tables for Investigators and Clinicians. Gastroenterology. 2016:S0016-5085(16)00180-3. doi: 10.1053/j. gastro.2016.02.014.

15. Qureshi SR, Abdelaal AM, Janjua ZA, Alasmari HA, Obad AS, Alamodi A, Shareef MA. Irritable Bowel Syndrome: A Global Challenge Among Medical Students. Cureus. 2016;8:e721. doi: 10.7759/cureus.721.

16. Al-Ghamdi S, AlOsamey F, AlHamdan A, Alnujaydi A, Turkistani A, AlRasheed A, et al. A study of impact and prevalence of irritable bowel syndrome among medical students. Int J Med Med Sci. 2015;7:139-47. doi: 10.5897/IJMMS2015.1188

17. AlButaysh OF, AlQuraini AA, Almukhaitah AA, Alahmdi YM, Alharbi FS. Epidemiology of irritable bowel syndrome and its associated factors in Saudi undergraduate students. Saudi J Gastroenterol. 2020;26:89-93. doi: 10.4103/sjg.SJG_459_19.

18. Costanian C, Tamim H, Assaad S. Prevalence and factors associated with irritable bowel syndrome among university students in Lebanon: findings from a cross-sectional study. World J Gastroenterol. 2015;21:3628-35. doi: 10.3748/wjg. v21.i12.3628.

19. Wells M, Roth L, McWilliam M, Thompson K, Chande N. A cross-sectional study of the association between overnight call and irritable bowel syndrome in medical students. Can J Gastroenterol. 2012;26:281-4. doi: 10.1155/2012/865915.

20. Hori K, Matsumoto T, Miwa H. Analysis of the gastrointestinal symptoms of uninvestigated dyspepsia and irritable bowel syndrome. Gut Liver. 2009;3:192-6. doi: 10.5009/ 
gnl.2009.3.3.192.

21. Jemilohun AC, Abayomi O, Adebayo PB. Prevalence of irritable bowel syndrome, psychological ill-health and health-seeking behavior in a population of Nigerian Medical Students. J Adv Med Med Res. 2018;25:1-9. doi: 10.9734/JAMMR/2018/39235

22. Tan YM, Goh KL, Muhidayah R, Ooi CL, Salem O. Prevalence of irritable bowel syndrome in young adult Malaysians: a survey among medical students. J Gastroenterol Hepatol. 2003;18:1412-6. doi: 10.1046/j.1440-1746.2003.03212.x.

23. Canavan C, West J, Card T. The epidemiology of irritable bowel syndrome. Clin Epidemiol. 2014;6:71-80. doi: 10.2147/CLEP. S40245.

24. Keshteli AH, Dehestani B, Daghaghzadeh H, Adibi P. Epidemiological features of irritable bowel syndrome and its subtypes among Iranian adults. Ann Gastroenterol. 2015;28:253-258

25. Jahangiri P, Jazi MS, Keshteli AH, Sadeghpour S, Amini E, Adibi P. Irritable Bowel Syndrome in Iran: SEPAHAN Systematic Review No. 1. Int J Prev Med. 2012;3:S1-9.
26. Tang YR, Yang WW, Wang YL, Lin L. Sex differences in the symptoms and psychological factors that influence quality of life in patients with irritable bowel syndrome. Eur J Gastroenterol Hepatol. 2012;24:702-7. doi: 10.1097/ MEG.0b013e328351b2c2.

27. American College of Gastroenterology Task Force on Irritable Bowel Syndrome, Brandt LJ, Chey WD, Foxx-Orenstein AE, Schiller LR, Schoenfeld PS, et al. An evidence-based position statement on the management of irritable bowel syndrome. Am J Gastroenterol. 2009;104 Suppl 1:S1-35. doi: 10.1038/ ajg.2008.122.

28. Kim YJ, Ban DJ. Prevalence of irritable bowel syndrome, influence of lifestyle factors and bowel habits in Korean college students. Int J Nurs Stud. 2005;42:247-54. doi: 10.1016/j. ijnurstu.2004.06.015.

29. Ibrahim NK. A systematic review of the prevalence and risk factors of irritable bowel syndrome among medical students. Turk J Gastroenterol. 2016;27:10-6. doi: 10.5152/ tjg.2015.150333. 